\title{
Predictors and Reasons of Permanent Tooth Extraction Among High School Children: A National Cross-Sectional study
}

\author{
Ammar A Almarghlani ${ }^{1}$, A Shatha Alshali ${ }^{2}$, Mohammad Assaggaf ${ }^{1}$, Ali S Alghamdi ${ }^{3}$, Mohammed \\ Alzahrani $^{3}$ \\ ${ }^{1}$ Assistant professor, Department of Periodontics, Faculty of Dentistry, King Abdulaziz University, Jeddah, Saudi Arabia. \\ 2 Assistant professor, Department of Prosthodontics, Faculty of Dentistry, King Abdulaziz University, Jeddah, Saudi Arabia \\ 3 Professor, Department of Periodontics, Faculty of Dentistry, King Abdulaziz University, Jeddah, Saudi Arabia
}

*Corresponding Author: Dr. Ammar Almarghlani, Assistant professor, department of Periodontics, Faculty of Dentistry, King Abdulaziz University, PO Box 109725, Jeddah 21351, Kingdom of Saudi Arabia. Tel. +966 (2) 6402000 Ext. 21206. Fax. +966 (12) 6404183. E-mail: ammar.marg@live.com

\begin{abstract}
Background: Tooth loss is a major dental health concern that has adverse consequences on the remaining dentition and on the patients' general wellbeing. The present study aimed to assess predictors and causes of permanent tooth extraction among school students. Methods: This national cross- sectional study included school children and spanned from September 2012 to January 2016. Demographic, social, and medical history recorded. Periodontal and dental examinations were performed. Multivariable logistic regression was used to determine predictors of tooth loss among the sample. Results: A total of 2435 school children were included in the study. Twenty-four percent of the students had extraction of at least one permanent tooth. Almost $27 \%$ of the female students had a permanent tooth extraction compared to only $21.7 \%$ of the male students, which was statistically significant. Students who visited dentists regularly had significantly more tooth extraction $(39 \%)$ than student who did not $(20.6 \%)$. Multivariate logistic regression analysis showed significant predictors for permanent tooth extraction were age, regular dental visits and mean probing depth (PD). Caries (15\%) followed by orthodontic treatment (6\%) were the main reason for permanent tooth extraction among the sample. Conclusion: Caries was responsible for majority of tooth loss among the study population. Significant predictors for permanent tooth extraction were age, regular dental visits and mean probing depth. There is a need for intensified oral health education and awareness programs in the population with emphasis on prevention of dental caries.
\end{abstract}

Keywords: Tooth loss, Permanent tooth extraction, Cross-sectional study.

\section{INTRODUCTION}

Tooth loss is a major dental health concern that has adverse consequences on the remaining dentition and on the patients' general wellbeing [1]. It could result from caries and its sequelae, periodontal disease, trauma, infection or malignancies [2]. However, this may differ from one patient to another depending on socioeconomic and educational levels [3]. Failure to replace missing tooth may affect balance in the stomatognathic system and trigger chains of adverse effects [4]. This may include masticatory problems and chewing difficulties, altered speech, loss of self-confidence, concern about appearance, and feelings of bereavement [5-7]. Tooth loss may also lead to rotation and drifting of adjacent teeth and supra-eruption of opposing teeth, which in turn may result in facial asymmetry or bite collapse. Tooth loss prevention strategy is essential for esthetic, occlusal functions, socio-economical and psychological reasons. Dental and periodontal diseases are epidemic and widespread health problems in many nations, especially among developing countries ${ }^{[8]}$, which can be prevented and treated if diagnosed early. In these countries, restoring natural tooth may be costly and extraction may be an inexpensive alternative in terms of finances and time ${ }^{[9]}$. Caries is the main cause of tooth loss in developed countries, such as Canada, Finland, New Zealand, Scotland, France, Norway, Sri Lanka, Sweden and 
Malaysia. Periodontal diseases, including periodontitis and gingivitis, and caries which have been reported to be high in the Saudi population, and in developing countries (e.g. India) [10, 11], are the main cause of dental extractions. However, caries and periodontal disease seem to cause almost equal percentages of tooth loss, in another group of countries, such as the United States [12]. Patterns of oral diseases and management are changing: a shift in emphasis from extraction to prevention as of the incidence dental caries is decreasing, aiming to preserve as much tooth structure as possible. This shift may have an influence on reasons of the tooth extraction. Despite improvement in different prevention and operative modality, tooth extraction remains an important treatment option [13]. Tooth loss may be also due to trauma, and prosthodontic reasons. Another reason for tooth extractions is orthodontic treatment. For instance, Ng'ang'a reported that orthodontic treatment accounted for $13 \%$ of extractions in Nairobi population [14]. Research to identify reasons for tooth extractions have been carried out in many countries and mainly in adult populations [15]. There have been no recent studies on the prevalence of tooth loss and reasons for tooth extractions in school children subjects in Saudi Arabia.

The present study aimed to assess prevalence, predictors and reasons of permanent tooth extraction among school students and to synthesize data on the association between various predictors and tooth extraction.

\section{MATERIALS AND METHODS}

This study was a descriptive cross-sectional to assess the predictors and reasons of permanent tooth extraction among school children in Saudi Arabia. Ethical approval was obtained. A random sample of school children grades 10 to 12 (15-18 years old) of both genders were included. The study spanned from September 2012 to January 2016. A "Multistage Clustered Sampling Design" was employed and was reported in detail in an earlier report [11]. The total number student in the selected cities were: Riyadh ( $n=713)$, Jeddah $(n=657)$, Dammam $(n=506)$, Abha $(n=297)$ and Tabuk $(n=262)$. Informed consent was sent to the parents of children to be included into the study to sign. The study aimed at sampling a randomly selected sample of, healthy school children and school children with medical conditions that do not have correlation with periodontitis, grades 10 to 12 (15-19 years old) of both genders. Students or parents refused to sign the consent or rejected the periodontal examination, and student with medical conditions that have been reported to have relation with periodontitis were excluded from the study. No children were admitted to the study without their parents' approval. Subject name, gender, age, address, and socioeconomic status were recorded. At examination visit, the examiners reviewed the medical and dental histories with the students and confirmed by their parents recorded the information. A dental history included history and causes of permanent tooth extraction. Periodontal and dental examinations were performed on permanent teeth only; primary teeth (if present), partially erupted teeth and third molars were excluded from the examination. The detailed examination was reported in an earlier study. The gingival and periodontal examination consisted of measurement of the gingival and periodontal supporting tissue including attachment loss, probing depth using Williams probe and furcation involvement; and the assessment of tooth mobility, gingival bleeding, dental plaque, dental calculus, and gingival recession.

\section{Statistical Methodology}

This study was analyzed using IBM SPSS ${ }^{\text {тM }}$ 22.0.0 (@SPSS Inc., USA). A simple descriptive statistic used to define the characteristics of the study sample variables through a form of counts and percentages for the categorical and nominal variables, while mean and standard deviation used to present the continuous variables. To test the bivariate relationship between tooth loss and categorical and continuous variables, this study used chi-square and independent t-test, respectively. These tests were done with the assumption of normal distribution. Multivariable logistic regression was used to determine predictors of tooth loss among the study sample. A model with backward conditional elimination with enter criterion $=0.05$ and elimination $=0.10$ was utilized. Statistical significance was set at $P<.05$

\section{RESULTS}

Table 1 shows the bivariables association between study samples' characteristics and previous permanent tooth extracted. It shows that $24 \%$ of the students had extraction of at least one permanent tooth. Almost $27 \%$ of the female students had a permanent tooth extraction compared to only $21.7 \%$ of the male students, which was statistically significant. Almost $28 \%$ of smokers had history of tooth loss while only $23 \%$ of non-smoker lost permanent tooth which was not statically significant. Students who visited dentists regularly had significantly more tooth extractions (39\%) than student who did not (20.6\%). In terms of brushing frequency, the more students brush their teeth the more extraction of permanent tooth was found. Students with history of periodontal diseases showed significantly more extracted permanent teeth than students with healthy periodontium (30\% and $23 \%$ respectively). Percentages of clinical attachment loss $(\mathrm{CAL}) \geq 1 \mathrm{~mm}$ have a significant relationship with previous permanent tooth extracted. Table 2 shows the results of the multivariable logistic regression models. Controlling for other variables, significant predictors for permanent tooth extraction were age, regular dental visits and mean pocket depth (PD). Increased age, more regular dental visits and increased PD were associated with more tooth extraction.

Table 3 shows the causes of tooth extraction among the students. It shows that the majority of the sample $(n=2435)$ extracted a permanent tooth due caries $(15 \%$ of the total sample) followed by orthodontic treatment $(6 \%$ of the total sample). Only $1 \%$ of the students extracted permanent tooth due to periodontal diseases (mobility) and another $1 \%$ due to trauma. Among those who had tooth extraction $(n=560)$, caries was responsible for $63 \%$ of the extractions whereas periodontal disease was responsible for only $6 \%$ as shown in figure 1 . 
Table 1: Characteristics of the 2435 Study Samples Relative to Previous Permanent Teeth Extracted

\begin{tabular}{|c|c|c|c|c|}
\hline \multirow{2}{*}{\multicolumn{2}{|c|}{ Variables }} & \multicolumn{2}{|c|}{ Previous Permanent Teeth Extracted } & \multirow[t]{2}{*}{$P$-value } \\
\hline & & Yes & No & \\
\hline \multicolumn{2}{|l|}{ Total N(\%) } & $585(24.0)$ & $1850(76.0)$ & $\mathrm{N} / \mathrm{A}$ \\
\hline \multicolumn{2}{|l|}{ Age Mean \pm SD } & $17.38 \pm 1.0$ & $17.22 \pm 1.0$ & $0.02^{\mathrm{b}}$ \\
\hline \multirow[t]{2}{*}{ Nationality N(\%) } & Non Saudi & $49(25.0)$ & $147(75.0)$ & \multirow[t]{2}{*}{0.73} \\
\hline & Saudi & $536(23.9)$ & $1703(76.1)$ & \\
\hline \multirow[t]{2}{*}{ Gender N(\%) } & Male & $288(21.7)$ & $1041(78.3)$ & \multirow[t]{2}{*}{$0.03^{a}$} \\
\hline & Female & $297(26.9)$ & $809(73.1)$ & \\
\hline \multirow[t]{2}{*}{ Smoker N(\%) } & Yes & $56(27.9)$ & $145(72.1)$ & \multirow[t]{2}{*}{0.18} \\
\hline & No & $529(23.7)$ & $1705(76.3)$ & \\
\hline \multirow[t]{2}{*}{ Regular Dental Visits N(\%) } & Yes & 178(39.1) & $277(60.9)$ & \multirow[t]{2}{*}{$<0.01^{\mathrm{a}}$} \\
\hline & No & $407(20.6)$ & 1573(79.4) & \\
\hline \multirow[t]{2}{*}{ Brushing $\mathrm{N}(\%)$} & Yes & $530(24.6)$ & $1626(75.4)$ & \multirow[t]{2}{*}{0.73} \\
\hline & No & $55(19.7)$ & $224(80.3)$ & \\
\hline \multirow[t]{3}{*}{ Brushing Frequency N(\%) } & Once & $200(22.8)$ & $678(77.2)$ & \multirow[t]{3}{*}{$0.04^{\mathrm{a}}$} \\
\hline & Twice & $218(23.6)$ & $704(76.4)$ & \\
\hline & More than 2 times & $112(31.5)$ & $244(68.5)$ & \\
\hline \multirow[t]{2}{*}{ Flossing $\mathrm{N}(\%)$} & Yes & $67(26.3)$ & $188(73.7)$ & \multirow[t]{2}{*}{0.37} \\
\hline & No & $518(23.8)$ & 1662(76.2) & \\
\hline \multirow[t]{2}{*}{ Tongue Brushing N(\%) } & Yes & $216(24.8)$ & $655(75.2)$ & \multirow[t]{2}{*}{0.50} \\
\hline & No & $369(23.6)$ & $1195(76.4)$ & \\
\hline \multirow[t]{2}{*}{ Previous Dental Treatment N(\%) } & Yes & $540(32.6)$ & $1117(67.4)$ & \multirow[t]{2}{*}{$<0.01^{\mathrm{a}}$} \\
\hline & No & $45(5.8)$ & $733(94.2)$ & \\
\hline \multirow[t]{2}{*}{ Periodontal Disease N(\%) } & No & $522(23.5)$ & 1704(76.5) & \multirow[t]{2}{*}{$0.03^{a}$} \\
\hline & Yes & $63(30.1)$ & 146(69.9) & \\
\hline \multicolumn{2}{|l|}{ Missing Teeth Mean \pm SD } & $1.05 \pm 1.4$ & $0.20 \pm 0.7$ & $<0.01^{c}$ \\
\hline \multicolumn{2}{|l|}{ Mean PD Mean \pm SD } & $0.65 \pm 0.2$ & $0.57 \pm 0.2$ & $<0.01^{\mathrm{c}}$ \\
\hline \multicolumn{2}{|l|}{ Mean CAL Mean \pm SD } & $0.07 \pm 0.2$ & $0.04 \pm 0.1$ & $0.02^{\mathrm{c}}$ \\
\hline \multicolumn{2}{|l|}{ PD $(\%) \geq 4 \mathrm{~mm}$ Mean \pm SD } & $2.16 \pm 5.2$ & $1.76 \pm 4.7$ & 0.10 \\
\hline \multicolumn{2}{|l|}{ CAL $(\%) \geq 1 \mathrm{~m}$ Mean \pm SD } & $3.53 \pm 13.2$ & $2.22 \pm 10.3$ & $0.03^{c}$ \\
\hline
\end{tabular}

Table 2: Binary Logistic Regression Model of Characteristics of the 2435 Study Samples Relative to Previous Permanent Teeth Extracted

\begin{tabular}{|c|c|c|c|c|}
\hline \multirow[t]{2}{*}{ Variables in the Equation } & \multirow[t]{2}{*}{$\overline{O R}$} & \multicolumn{2}{|c|}{ 95\% C.I. for EXP(OR) } & \multirow[t]{2}{*}{$P$-value } \\
\hline & & Lower & Upper & \\
\hline Age & 1.2 & 1.3 & 1.0 & $0.016^{\mathrm{b}}$ \\
\hline Smoker (Yes vs No) ${ }^{\mathrm{C}}$ & 1.5 & 2.2 & 1.0 & 0.054 \\
\hline Regular Dental Visits (Yes vs No) $^{c}$ & 1.4 & 2.0 & 1.17 & $0.002^{\mathrm{b}}$ \\
\hline 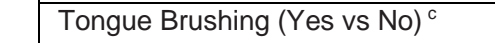 & 0.78 & 1.0 & 0.60 & 0.053 \\
\hline Gingival Index & 1.16 & 1.3 & 1.0 & 0.086 \\
\hline Mean PD & 3.84 & 8.33 & 1.72 & $0.001^{\mathrm{b}}$ \\
\hline Constant & 687.090 & & & $<0.001^{\mathrm{b}}$ \\
\hline
\end{tabular}

b-Significant using Binary Logistic Regression Model, with Backward Conditional Elimination with Enter Criterion=0.05, Elimination=0.10. Model fit measures. -2 Log likelihoods: 2271.325, AIC: 2285.325, R2 (Cox \& Snell): 0.066, R2 (Nagelkerke): 0.099.

${ }^{\mathrm{C}}$ No is the reference 
Table 3: Reasons for history of permanent tooth extraction among the study sample

\begin{tabular}{|l|l|l|}
\hline \multicolumn{3}{|c|}{ Previous Permanent Teeth Extracted } \\
\hline Reason for tooth extraction & \multicolumn{1}{|c|}{ Yes } \\
\hline Caries N(\%) & $354(15)$ & $2018(85)$ \\
\hline Orthodontic treatment N(\%) & $151(6)$ & $2284(94)$ \\
\hline Mobility N(\%) & $34(1)$ & $2401(99)$ \\
\hline Trauma N(\%) & $21(1)$ & $2414(99)$ \\
\hline
\end{tabular}

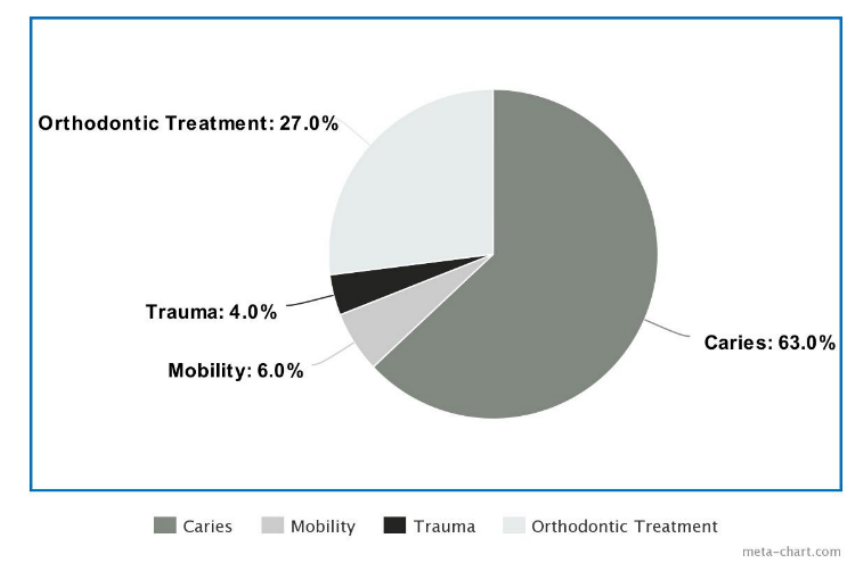

\section{DISCUSSION}

Tooth loss can provide information regarding the availability of dental care, the prevalence of dental disease, and attitudes toward tooth loss. In the present study, $24 \%$ of the students had extraction of at least one permanent tooth, while it was $40.9 \%$ in cross sectional study that was carried out in the Eastern Province of Saudi Arabia on same age group [11, 16].

Despite numerous previous studies having examined the nature of tooth loss and dental extractions of permanent tooth in adults, surprisingly little information exists regarding loss of permanent tooth among school children [17]. One must be cautious in interpreting the comparison of studies' results because of cultural and demographic differences and the availabilities of dental services in different countries. When comparing results of independent studies carried out at different points of time care must be taken since various sources of bias may invalidate the comparisons ${ }^{[18]}$.

The present study shows the tendency of more extraction of permanent tooth among females than males, and this is consistent with some earlier studies [19-21]. Other studies, however, showed a greater percentage of permanent tooth extraction among males than females ${ }^{[12,22,23]}$; but these studies sampled different age groups than the present study. The increased tooth extractions among females could be due to more frequent dental visits for management of inadequate and unaesthetic dentition rather than being a true reflection of higher prevalence of tooth mortality than the males ${ }^{[7,24]}$. It is important to note that attending regular dental visits is a very important protective factor against periodontal diseases. Since the present study was conducted on children, who have a lower prevalence of destructive periodontal disease than adults, it showed that students who visited dentists regularly and brush their teeth more frequently had significantly more extraction of permanent tooth than students who did not. In addition, some of these extractions may be due to financial issues, as restoring tooth is more expensive and time consuming than extracting them [25]. Another reason contributing to these results could be due to the need for permanent tooth extraction for therapeutic reasons (i.e. orthodontic treatment), which was the second most common reason for tooth extraction among the students. Nevertheless, the importance of attending regular dental visits must be emphasized to allow for early detection and treatment of emerging oral diseases, including periodontal conditions, and for evaluation and reinforcement of oral hygiene measures.

Regarding extraction of permanent tooth, several conclusions were obvious from the data. First of all, caries was the most common reason for extraction. These results are consistent with a study conducted in the US, which reviewed 2000 records and concluded that $53 \%$ of tooth extraction was mainly due to caries and pulpal pathosis [17]. Another study reported that caries accounted for $81 \%$ of tooth extraction in UAE [26].

In the present study, tooth extractions due to periodontitis were found in only $1 \%$ of the sample. This finding is consistent with a study on an Italian sample that reported that no one under 20 years of age had extractions due to periodontal diseases [27]. These findings contradict a study that included Sudanese subjects aged older than 17 years, which reported that periodontitis accounted for $34.8 \%$ of tooth loss. The difference in the presented data could be due to the cultural differences or variations in the socioeconomic status and accessibility to dental care between the samples ${ }^{[28]}$.

Prevalence of malalignment, mainly crowding, and need for orthodontic treatment among the Saudi population were reported to be high [29]. Therefore, not surprisingly, tooth extraction for orthodontic reasons was the second main reason for permanent tooth extraction among the study sample ${ }^{[30]}$. These results are consistent with another study that included a Saudi sample ${ }^{[20]}$.

The present study indicated that caries is the principle reason for tooth extraction among the school children in the studied Saudi Arabian population, followed by extraction for orthodontic reasons. Therefore, caries-prevention measures must begin early in life among this population to identify those at risk. There is the need for intensified oral health education and awareness programs in the population with emphasis on prevention of dental caries and early dental intervention in order to avoid premature tooth loss in children and adolescents. Certainly, this study and many others support the need for further studies defining these risk factors and exploring methods of intervention.

\section{CONCLUSION}

The present study indicated that caries is the principle reason 
for tooth extraction among the school children in the studied Saudi Arabian population, followed by extraction for orthodontic reasons. Therefore, caries-prevention measures must begin early in life among this population to identify those at risk. Certainly, this study and many others support the need for further studies defining these risk factors and exploring methods of intervention in those at risk.

Why this paper is important to paediatric dentists

1- This paper indicates the reasons and predictors of permanent tooth loss among high school children.

2- It involved large sample size (2435) and it's a national study included different regions of Saudi Arabia.

3- It is important to know the predictors of tooth loss to formulate large educational and preventive programs.

\section{REFERENCES}

1. Kumar S, Gupta P, Gupta V, Gupta B. Evaluation of clinical consequences postpartial edentulism in patients of Ranchi District: An epidemiological study. J Dent Res Rev. 2018; 5:84-7.

2. Holt R, Roberts G, Scully C. Dental damage, sequelae, and prevention. West J Med. 2001; 174(4):288-90.

3. Park JB, Han K, Park YG, Ko Y. Association between socioeconomic status and oral health behaviors: The 2008-2010 Korea national health and nutrition examination survey. Exp Ther Med. 2016; 12(4):2657-64.

4. Rosenstial S, Land M, Fiyimoto J. Contempory fixed prosthodontics. St. Louis: Mosby. 1995.

5. Krall E, Garvey A, Garcia R. Alveolar bone loss and tooth loss in male Cigar and pipe smokers. J Am Dent Association. 1999; 130:57-64.

6. Burt B, Eklund S. Dentistry, Dental Practice and the community. 5th ed. Philadelphia: W. B. Saunders C;. 2005; 203-11.

7. Vigu A, Stanciu D. When the fear of dentist is relevant for more than one's oral health. A structural equation model of dental fear, self-esteem, oral-health-related well-being, and general wellbeing. Patient Prefer Adherence. 2019; 13:1229-40.

8. Nazir MA. Prevalence of periodontal disease, its association with systemic diseases and prevention. Int $\mathrm{J}$ Health Sci (Qassim). 2017; 11(2):72-80.

9. Dixit L, Gurung C, Gurung N, Joshi N. Reasons underlying the extraction of permanent teeth in patients attending Peoples Dental College and Hospital. Nepal Med Coll J. 2010; 12:203-06.

10. al Shammery A, el Backly M, Guile E. Permanent tooth loss among adults and children in Saudi Arabia. Community Dent Health. 1998; 15(4):277-80.

11. AlGhamdi A, Almarghlani A, Alyafi R, Ibraheem W, Assaggaf M, Howait $M$, et al. Prevalence of periodontitis in high school children in Saudi Arabia: a national study. Ann Saudi Med. 2020; 40(1):714.

12. Farsi J. Common causes of extraction of teeth in Saudi Arabia. Saudi Dent J. 1992; 4(3):101-5.

13. Southerland JH, Gill DG, Gangula PR, Halpern LR, Cardona CY, Mouton CP. Dental management in patients with hypertension: challenges and solutions. Clin Cosmet Investig Dent. 2016; 8:11120.

14. Ng'ang'a P. A study of occlusal anomalies and tooth loss in children aged 13-15 years in Nairobi. East Afr Med J. 1991; 68:980-88.

15. Aida J, Ando Y, Akhter R, Aoyama H, Masui M, Morita M. Reasons for permanent tooth extractions in Japan. J Epidemiol. 2006; 16(5):214-9.

16. Atieh MA. Tooth loss among Saudi adolescents: social and behavioural risk factors. Int Dent J. 2008; 58(2):103-8.

17. Alsheneifi $T$, Hughes $C$. Reasons for dental extractions in children. Pediatr Dent. 2001; 23(2):109-12.

18. Agerholm D. Reasons for extraction by dental practitioners in England and Wales: a comparison with 1986 and variations between regions. J Dent. 2001; 29:237-41.

19. Shinawi LA. Partialedentulism: a five year survey on the prevalence and pattern of tooth loss in a sample of patients attending King Abdul Aziz University - Faculty of Dentistry. Life SciJ. 2012; 94:2665-71.

20. Alesia $\mathrm{K}, \mathrm{Khalil} \mathrm{H}$. Reasons for and patterns relating to the extraction of permanent teeth in a subset of the Saudi population. Clin Cosmet Investig Dent 2013; 30(5):51-6.

21. Axell T OB. Prevalence of removable dentures and edentulism in adult Swedish population. Swed Dent J. 1997; 3:129-37.

22. Muneeb A, Khan B, Jamil B. Causes and pattern of partial edentulism/exodontia and its association with age and gender: semi-rural population, Baqai dental college, Karachi, Pakistan. Inter Dent J Stud Res. 2013; 1(3):13-8.

23. Thomas S, Al-Maqdassy S. Causes and Pattern of Tooth Mortality among Adult Patients in a Teaching Dental Hospital. Bnosina. J Med Biomed Sci. 2010; 2(4):160-7.

24. Renson C, Crielaers P, Ibikunle S, Pinto V, Ross C, Infirri J, et al. Changing patterns of oral health and implications for oral health manpower. Part I. Report of a working group convened jointly by the Federation Dentaire Internationale and the WHO. Int Dent J. 1985; 35:235-51.

25. Avila G, Galindo-Moreno P, Soehren S, Misch C, Morelli T, Wang $\mathrm{H}$. A novel decision-making process for tooth retention or extraction. J Periodontol. 2009; 80(3):476-91.

26. Hashim R, Salah A, Hamid A, Hamad A, Slieby K. Reasons for permanent teeth extraction in the United Arab Emirates. J Int Oral Health. 2019; 11:304-8.

27. Angelillo I, Nobile C, Pavia M. Survey of reasons for extraction of permanent teeth in Italy. Community Dent Oral Epidemiol. 1996; 24:336-40.

28. Yousaf A MS, Yousaf N, Bangash K, Manzoor M. Reasons for extractions in patients seen in PAK field hospital Level 3 Darfur, Sudan. Pakistan Oral Dent J. 2012; 2012(32):393-6.

29. al-Emran S, Wisth P, Böe O. Prevalence of malocclusion and need for orthodontic treatment in Saudi Arabia. . Community Dent Oral Epidemiol. 1990; 18(5):253-5.

30. Fatani NH, Hammam MB, Oraif H, Taher S, Taju W, Bukhari O. Prevalence of Malocclusion among Schoolchildren in Makkah, Saudi Arabia. Open Access Maced J Med Sci. 2019; 7(5):856-61. 